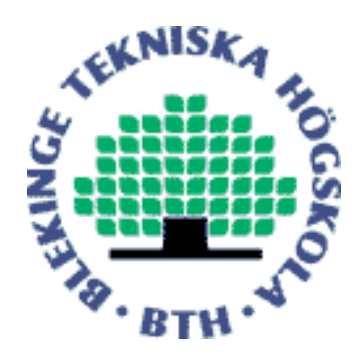

Electronic Research Archive of Blekinge Institute of Technology http://www.bth.se/fou/

This is an author produced version of a paper published in Proceedings of the Eighth International Conference on Artificial Intelligence and Soft Computing - ICAISC 2006, June, 2006, Zakopane, Poland. This paper has been peer-reviewed but may not include the final publisher proof-corrections or journal pagination.

Citation for the published paper: Elisabeth Rakus-Andersson.

The Greatest and the Least Eigen Fuzzy Sets in Evaluation of the Drug Effectiveness Levels.

Lecture Notes in Computer Science - Springer-verlag, 2006, Vol. 4029/2006, pp 982-988/ICAISC 2006, June 2006, Zakopane, Poland. 


\title{
The Greatest and the Least Eigen Fuzzy Sets in Evaluation of the Drug Effectiveness Levels
}

\author{
Elisabeth Rakus-Andersson \\ Blekinge Institute of Technology, School of Engineering, Study of Mathematics, \\ S-37179 Karlskrona, Sweden \\ Elisabeth. Anderssonabth. se
}

\begin{abstract}
The eigen fuzzy set of a given fuzzy relation often corresponds to an occurrence of invariability in natural sciences. By determining the fuzzy relations as connections between pairs of symptoms we utilize the greatest and the least eigen fuzzy sets in order to find the estimates of the medicine effectiveness levels.
\end{abstract}

\section{Introduction}

The existence of the greatest eigen fuzzy set of a fuzzy relation was confirmed in the 80 -ties of the twentieth century $[4,7,8,9]$. In the latest investigations the scientists have proved that even the least eigen fuzzy set can be generated for the given relation $[1,2,5]$. The eigen fuzzy sets have already been applied to the evaluation of medicine action levels when considering the medicine influence on clinical symptoms $[3,6]$.

We continue the last item by accomplishing an own proof of the existence of the least set especially, which differs from the conceptions formulated in $[1,2,5]$. The theoretical discussion, which concerns eigen sets, constitutes the contents of Section 2. In Section 3 we introduce the medical problem that involves applications of eigen fuzzy sets. Finally, a simple medical exercise is solved in Section 4 to give an image of the functional utility of the presented model.

\section{Theoretical Assumptions of Eigen Fuzzy Problem}

A particular result of a relation composition is known as the eigen set of a fuzzy relation $[7,8]$.

Assume that $X=\left\{x_{1}, \ldots, x_{n}\right\}$ is a finite set of real numbers. The eigen fuzzy set of the fuzzy relation $R \subseteq X \times X$ is a set $A \subseteq X$, which satisfies $R \circ A=A$.

$R$ is the fuzzy relation determined as $R \subseteq X \times X$ with the membership function $\mu_{R}\left(x, x^{\prime}\right): X \times X \rightarrow[0,1], x, x^{\prime} \in X$. It is proved that the eigen fuzzy set $A \subseteq X$, $\mu_{A}(x): X \rightarrow[0,1]$, which is a part of the equation $A \circ R=A$, exists [7, 8, 9].

We define the set $A_{0}$ with $\mu_{A_{0}}(x)=a_{0}, a_{0}=\min _{x^{\prime} \in X}\left(\max _{x \in X} \mu_{R}\left(x, x^{\prime}\right)\right)$ for all $x \in X$. 
The fuzzy connection $A_{0} \circ R=A_{0}$ is a true equality because of $\mu_{A_{0} \circ R}\left(x^{\prime}\right)=$ $\max _{x}\left(\min \left(\mu_{A_{0}}(x), \mu_{R}\left(x, x^{\prime}\right)\right)=\max _{x}\left(\min \left(a_{0}, \mu_{R}\left(x, x^{\prime}\right)\right)=\min \left(a_{0}, \max _{x} \mu_{R}\left(x, x^{\prime}\right)\right)=a_{0}=\right.\right.$ $\mu_{A_{0}}\left(x^{\prime}\right), \quad x, x^{\prime} \in X$. Hence, $A_{0}$ is an eigen fuzzy set of $R$.

The next introduced set $A_{1}$ is identified by its membership function given by

$$
\mu_{A_{1}}\left(x^{\prime}\right)=\max _{x \in X} \mu_{R}\left(x, x^{\prime}\right)
$$

for all $x^{\prime} \in X$.

The fuzzy sets, which are members of the sequence $\left(A_{n}\right)_{n}$, in which

$$
A_{2}=A_{1} \circ R=A_{1} \circ R^{1}, A_{3}=A_{2} \circ R=A_{1} \circ R^{2}, \cdots, A_{n+1}=A_{n} \circ R=A_{1} \circ R^{n},
$$

exist for all integers $n>0$.

The sets satisfy the inclusions

$$
A_{0} \subseteq \cdots \subseteq A_{n+1} \subseteq A_{n} \subseteq \cdots \subseteq A_{2} \subseteq A_{1} .
$$

To prove (3) we apply the mathematical induction. On the basis of the definition of $A_{0}$ we conclude that $A_{0} \subseteq A_{1}$ since $\left.\mu_{A_{0}}\left(x^{\prime}\right)=\min _{x^{\prime}}\left(\max _{x} \mu_{R}\left(x, x^{\prime}\right)\right) \leq \max _{x} \mu_{R}\left(x, x^{\prime}\right)\right)$ $=\mu_{A_{1}}\left(x^{\prime}\right)$. We deduce that even $A_{2} \subseteq A_{1}$. By conveying, for every $x^{\prime} \in X$, that $\mu_{A_{2}}\left(x^{\prime}\right)=\mu_{A_{1} \circ R}\left(x^{\prime}\right)=\max _{x \in X}\left(\min \left(\mu_{A_{1}}(x), \mu_{R}\left(x, x^{\prime}\right)\right) \leq \max _{x \in X} \mu_{R}\left(x, x^{\prime}\right)=\mu_{A_{1}}\left(x^{\prime}\right)\right.$, we state that $A_{2} \subseteq A_{1}$.

We shall now prove that the assumption $A_{n} \subseteq A_{n-1}$ induces the conclusion $A_{n+1} \subseteq A_{n}, n \in N$, since $A_{n} \subseteq A_{n-1} \rightarrow A_{n} \circ R \subseteq A_{n-1} \circ R \leftrightarrow A_{n+1} \subseteq A_{n}$.

The set $A_{0}$ is the eigen set of $R$. $A_{1}$, the other set proposed by (1), rarely is a solution of the restriction $A_{1} \circ R=A_{1}$. If $A_{n} \circ R=A_{n}$, for $A_{n}$ being a member of the sequence of sets given by (2), then we will allege that $A_{n}$ is the expected greatest eigen set of the relation $R$, which often differs from $A_{0}$. The set $A_{0}$ is the least set in the chain of sets in (2) and all sets included between $A_{1}$ and $A_{n}$ are not eigen.

Suppose that $A_{0} \neq A_{n+k}=\cdots=A_{n+1}=A_{n} \neq \cdots \neq A_{2} \neq A_{1}$, then the composition $A_{n} \circ R$ leads to $A_{n} \circ R=A_{1} \circ R^{n-1} \circ R=A_{1} \circ R^{n}=A_{n+1}=A_{n}$.

$A_{n}$ is thus the greatest eigen fuzzy set (GEFS) of $R$ provided that $A_{n}=A_{n+1}$.

We recall that membership degrees of $A_{n+1}$ are calculated as

$$
\mu_{A_{n+1}}\left(x^{\prime}\right)=\mu_{A_{n} \circ R}\left(x^{\prime}\right)=\max _{x \in X}\left(\min \left(\mu_{A_{n}}(x), \mu_{R}\left(x, x^{\prime}\right)\right)\right.
$$

for each $x^{\prime} \in X$.

It can be desirable to find the smallest eigen fuzzy set of a given fuzzy relation as well. In spite of some accomplished investigations of the topic [1,2] let us propose the own contribution as the following proof of the least eigen set existence.

We define a new set $A_{0}$ with $\mu_{A_{0}}(x)=a_{0}$ for all $x \in X$, where

$a_{0}=\max _{x^{\prime} \in X}\left(\min _{x \in X} \mu_{R}\left(x, x^{\prime}\right)\right) . A_{0}$ is the eigen set of $R$ as it has been proved before. 
The set $A_{1}$ gets now new membership degrees determined as

$$
\mu_{A_{1}}\left(x^{\prime}\right)=\min _{x \in X} \mu_{R}\left(x, x^{\prime}\right)
$$

for all $x^{\prime} \in X$.

We propose the same sequence of fuzzy sets $\left(A_{n}\right)_{n}, \quad A_{2}=A_{1} \circ R=A_{1} \circ R^{1}$, $A_{3}=A_{2} \circ R=A_{1} \circ R^{2}, \cdots, A_{n+1}=A_{n} \circ R=A_{1} \circ R^{n}$, which fulfill inclusions

$$
A_{1} \subseteq A_{2} \subseteq \cdots \subseteq A_{n} \subseteq A_{n+1} \subseteq \cdots \subseteq A_{0}
$$

The boundary inclusion $A_{1} \subseteq A_{0}$ in the chain is true because of the inequality $\left.\mu_{A_{0}}\left(x^{\prime}\right)=\max _{x^{\prime}}\left(\min _{x} \mu_{R}\left(x, x^{\prime}\right)\right) \geq \min _{x} \mu_{R}\left(x, x^{\prime}\right)\right)=\mu_{A_{1}}\left(x^{\prime}\right)$.

To confirm the reliability of other inclusions in (6) we return to the assumptions of the mathematical induction. In order to check that $A_{1} \subseteq A_{2}$ we thus notice that $\mu_{A_{2}}\left(x^{\prime}\right)=\mu_{A_{1} \circ R}\left(x^{\prime}\right)=\max _{x \in X}\left(\min \left(\mu_{A_{1}}(x), \mu_{R}\left(x, x^{\prime}\right)\right) \geq \min _{x \in X} \mu_{R}\left(x, x^{\prime}\right)=\mu_{A_{1}}\left(x^{\prime}\right), x^{`} \in X\right.$.

The last structure is equivalent to $A_{1} \subseteq A_{2}$ since $\mu_{A_{1}}\left(x^{\prime}\right) \leq \mu_{A_{2}}\left(x^{\prime}\right)$.

The induction assumption $A_{n-1} \subseteq A_{n}$ is used in the proof to obtain the conclusion $A_{n} \subseteq A_{n+1}$. We start with $A_{n-1} \subseteq A_{n}$ to compose with $R$ both sides of the inclusion as $A_{n-1} \circ R \subseteq A_{n} \circ R$, which is comparable to $A_{n} \subseteq A_{n+1}$.

The set $A_{0}$ is the eigen set of $R$ but $A_{1}$ seldom is regarded as eigen. Let us assume that $A_{n}$ is a member of the sequence listed in (6) and that it fulfils $A_{n} \circ R=A_{n}$ for $A_{1} \neq A_{2} \neq \cdots \neq A_{n}=A_{n+1}=\cdots=A_{n+k} \neq A_{0}$. Then $A_{n}$ will be the least eigen set (LEFS) of the relation $R$, which is different from $A_{0}$.

In order to evaluate GEFS and LEFS we adopt a procedure, which consists of the following steps:

\section{Algorithm 1}

1. Find the set $A_{1}$,

2. Set the index $n=1$,

3. Calculate $A_{n+1}=A_{n} \circ R$,

4. $A_{n+1} \stackrel{?}{=} A_{n \rightarrow \text { Yes } \rightarrow A=A+A} \rightarrow \begin{gathered}\rightarrow \text { Go } \\ \text {, }\end{gathered}$ to step 3

in which $A_{1}$ is computed either by the application of (1) or by the choice of (5).

The relation $R$ keeps the given fuzzy set invariant, which apparently fits to a medical appearance when a medicine has no more effect in the curative process.

\section{Eigen Sets in Effectiveness Levels of Drugs}

Let us assume that characteristic qualitative symptoms of a morbid unit are found in a sample of patients. After the treatment some symptoms should disappear entirely while the other symptoms are still present. 
Let us denote a non-fuzzy set of symptoms by $S=\left\{S_{1}, \ldots, S_{n}\right\}$.

An estimation of the maximal level is possible by employing a fuzzy relation $R_{\max }$, which is created according to the formulation: "The action of the drug on the $j^{\text {th }}$ symptom is equal or stronger than on the $k^{\text {th }}$ one, $j, k=1, \ldots, n$ ". The membership degree $\mu_{R_{\max }}\left(S_{j}, S_{k}\right)$ indicates the strength of the relationship between the $j^{\text {th }}$ and the $k^{\text {th }}$ symptom.

If $m$ and $p$ denote the number of examined patients respectively the number of patients who fit for the definition of $R_{\max }$ when comparing the medication effects for $S_{j}$ and $S_{k}$, then we will compute the membership degrees $\mu_{R_{\max }}\left(S_{j}, S_{k}\right)$ as [3]

$$
\mu_{R_{\max }}\left(S_{j}, S_{k}\right)=\frac{p}{m}
$$

for $j, k=1, \ldots, n$.

Suppose that "-“ is assigned to the lack of a symptom after the treatment and "+" designates its presence in patient after the medication. The sign patterns "- -" and "+ ", counted with respect to the pair $S_{j}, S_{k}$ in the group of $m$ patients help us to appreciate $p$. For the pairs $\left(S_{j}, S_{j}\right), j=1, \ldots, n$, the value of $p$ is computed as a number of recoveries from $S_{j}$.

The relation $R_{\max }$ has the greatest eigen fuzzy set $A_{\max }$, which is defined in the universe $S$ as

$$
A_{\max } \circ R_{\max }=A_{\max } .
$$

$A_{\max }$ is found as the result of Algorithm 1, in which $A_{1}$ is defined by (1). The relation, designed in accordance with the statement: "The drug acts equally strongly or more strongly on the $j^{\text {th }}$ symptom than on the $k^{\text {th }}$ one" has its eigen set as an unchangeable component of the equation (8). We thus conclude that membership degrees of $A_{\max }$ show the level "the drug action on the considered symptoms is not stronger". Moreover, we are able to accept this level as optimal since $A_{\max }$ is the greatest solution of (8) in the sense of the greatest membership degree values.

An estimation of the minimal medicine effect is connected with forming another fuzzy relation $R_{\min }$ proposed as a clue:'The action of the drug on the $j^{\text {th }}$ symptom is equal to or weaker than on the $k^{\text {th }}$ one, $j, k=1, \ldots, n$." The suggested formula of calculating membership degrees of $R_{\min }$ is Eq. (7) to which the sign configurations "- -“ and "+-" are attached.

The relation $R_{\min }$ also generates its own, this time the least, eigen fuzzy set $A_{\min }$ that constitutes a compound of an equation

$$
A_{\min } \circ R_{\min }=A_{\min }
$$

To decide $A_{\min }$ we perform the steps of Algorithm 1, which includes $A_{1}$ computed by the action of (5).

$A_{\min }$ does not change its membership degrees after the next composition with $R_{\min }$ : "The drug affects equally or more weakly the $j^{\text {th }}$ symptom compared to the $k^{\text {th }}$ one". Then membership degrees of the least eigen set, associated with symptoms $S_{1}, \ldots, S_{n}$, indicate the minimal level of the medicine effectiveness. $A_{\min }$, as the least eigen set of $R_{\text {min }}$, provides us with the statement "the action of the medicine on the considered 
symptoms cannot be weaker".

The values of $\mu_{A_{\min }}\left(S_{j}\right)$ and $\mu_{A_{\max }}\left(S_{j}\right), j=1, \ldots, n$, constitute the borders of an interval, which is treated as the range of the medicine effectiveness for each symptom $S_{j}$. This should help us in making the judgement of the tested drug usability.

\section{The Medical Example}

The diagnosis $D$ known as a throat inflammation is accompanied by the set of symptoms $S=\left\{S_{1}=\right.$ "sore throat (pain)", $S_{2}=$ "temperature", $S_{3}=$ "inflammation state" $\}$. The physician has prescribed Bayer's aspirin as a remedy that should improve the health conditions in the group of 30 patients suffering from throat inflammation.

The application of (7) with the sign pattern "-_" and "- +" gives $A_{\max }$ as

$$
\begin{aligned}
& \begin{array}{lll}
S_{1} & S_{2} & S_{3}
\end{array}
\end{aligned}
$$

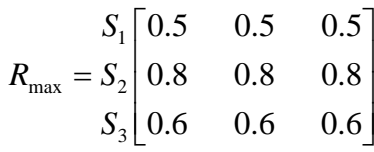

which has the greatest eigen fuzzy set decided as

$$
A_{\max }=\left[\begin{array}{ccc}
S_{1} & S_{2} & S_{3} \\
0.8 & 0.8 & 0.8
\end{array}\right] .
$$

Equation (7), in which numbers of the associations "- -" and "+ -" constitute the basis of the $p$ value computations, result in the relation $A_{\min }$ yielded as

$$
R_{\min }=S_{S_{2}}\left[\begin{array}{ccc}
S_{1} & S_{2} & S_{3} \\
S_{3}\left[\begin{array}{ccc}
0.5 & 0.8 & 0.6 \\
0.5 & 0.8 & 0.6 \\
0.5 & 0.8 & 0.6
\end{array}\right]
\end{array}\right.
$$

which possesses the least eigen fuzzy set

$$
\begin{array}{rcc}
S_{1} & S_{2} & S_{3} \\
A_{\min }=\left[\begin{array}{lll}
0.5 & 0.8 & 0.6
\end{array}\right] .
\end{array}
$$

By interpreting the membership degrees of $A_{\min }$ and $A_{\max }$ in the percentage scale we conclude that Bayer's aspirin removes $S_{1}$ in $50 \%-80 \%$ and $S_{2}-$ in $80 \%$, while $S_{3}$ disappears for $60 \%-80 \%$ of the sample of patients. 


\section{Conclusions}

As a counterpart of the discussion on the least eigen fuzzy set, we expand the own conception of the proof to confirm that the least eigen set exists.

The greatest and the least sets of fuzzy relations have been employed to approximate the optimal level of a medicine efficacious power. We have obtained the medicine action intervals evaluated for each symptom.

In constructing the relations we regard pairs of symptoms to learn about their influence on each other. Even if we appreciate effectiveness levels for individual symptoms, we will be aware of the complex dependency among symptoms, which influences single ranges. This aspect of complexity is an advantage of fuzzy research when comparing fuzzy results to computations of statistical ranges that do not consider interactions among the examined objects.

\section{References}

1. Bede, B., Nobuhara, H., Hirota, K.: Numerical Computation of Eigen Fuzzy Sets and Applications to Image Analysis. In: Dzitac, I., Maghiar, T., Popescu C. (eds.): Proc. of the International Conference on Computers and Communications, ICCC 2004. Univ. din Oradea, Romania (2004) 72-77

2. Fernández, M., Suárez, F., Gil, P.: T-eigen Fuzzy Sets, Inf. Sci. 75 (1993) 63-80

3. Gerstenkorn, T., Rakus, E.: An Application of Fuzzy Set Theory to Differentiating the Effectiveness of Drugs in Treatment of Inflammation of Genital Organs. Fuzzy Sets and Systems 68 (1994) 327-333

4. Goetschel, R., Voxman, W.: Eigen Fuzzy Number Sets. Fuzzy Sets and Systems 16 (1985) 75-85

5. Jacas, J., Recasens, J.: Fuzzy T-transitive Relations: Eigenvectors and Generators. Fuzzy Sets and Systems 72 (1995) 147-154

6. Rakus-Andersson E.: An Application of Fuzzy Numbers in Eigen Fuzzy Set Problem to Differentiating the Effectiveness of Drugs. In: Yinming Liu, Guoqing Chen (eds.): "Proc. of the International Conference on Fuzzy Information Processing - Theories and Applications. Tsinghua University Press - Springer (2003) 85-90

7. Sanchez, E.: Resolution of Eigen Fuzzy Set Equations. Fuzzy Sets and Systems 1 (1978) 69-74

8. Sanchez, E.: Eigen Fuzzy Sets and Fuzzy Relations. Journal of Mathematical Analysis and Applications 81 (1981) 399-421

9. Wagenknecht, M., Hartmann, K.: On the Construction of Fuzzy Eigen Solutions in Given Regions. Fuzzy Sets and Systems 20 (1986) 55-65 